\title{
The Shaker Type Potassium Channel, GORK, Regulates Abscisic Acid Signal- ing in Arabidopsis
}

\author{
Chae Woo Lim ${ }^{1}$, Sang Hee Kim², Hyong Woo Choi ${ }^{3 *}$, Sheng Luan ${ }^{4 *}$, and Sung Chul Lee ${ }^{1 *}$ \\ ${ }^{1}$ Department of Life Science (BK21 Program), Chung-Ang University, Seoul 06974, Korea \\ ${ }^{2}$ Division of Applied Life Science (BK 21 Plus Program), Plant Molecular Biology and Biotechnology Research Center, \\ Gyeongsang National University, Jinju 52828, Korea \\ ${ }^{3}$ Department of Plant Medicals, Andong National University, Andong 36729, Korea \\ ${ }^{4}$ Department of Plant and Microbial Biology, University of California, Berkeley, CA 94720, USA
}

(Received on July 18, 2019; Revised on August 28, 2019; Accepted on August 28, 2019)

Evolution of adaptive mechanisms to abiotic stress is essential for plant growth and development. Plants adapt to stress conditions by activating the abscisic acid (ABA) signaling pathway. It has been suggested that the ABA receptor, clade A protein phosphatase, SnRK2 type kinase, and SLAC1 anion channel are important components of the ABA signaling pathway. In this study, we report that the shaker type potassium $\left(\mathrm{K}^{+}\right)$channel, GORK, modulates plant responses to ABA and abiotic stresses. Our results indicate that the full length of PP2CA is needed to interact with the GORK Cterminal region. We identified a loss of function allele in gork that displayed $\mathrm{ABA}$-hyposensitive phenotype. gork and pp2ca mutants showed opposite responses to ABA in seed germination and seedling growth. Additionally, gork mutant was tolerant to the $\mathrm{NaCl}$ and mannitol treatments, whereas $p p 2 c a$ mutant was sensitive to the $\mathrm{NaCl}$ and mannitol treatments. Thus, our results indicate that GORK enhances the sensitivity to ABA and

\footnotetext{
*Corresponding author.

Hyong Woo Choi

Phone) +82-54-820-5509, FAX) +82-54-820-6320

Sheng Luan

Phone) +1-510-642-6306, FAX) +1-510-642-4995

Sung Chul Lee

Phone) +82-2-820-5207, FAX) +82-2-825-5206

ORCID

https://orcid.org/0000-0003-2725-0854

(c) This is an Open Access article distributed under the terms of the Creative Commons Attribution Non-Commercial License (http:// creativecommons.org/licenses/by-nc/4.0) which permits unrestricted noncommercial use, distribution, and reproduction in any medium, provided the original work is properly cited.
}

Articles can be freely viewed online at www.ppjonline.org. negatively regulates the mechanisms involved in high salinity and osmotic stresses via PP2CA-mediated signals.

Keywords : abscisic acid, high salinity, osmotic stress, PP2CA

Handling Editor : Jang, Cheol Seong

Global warming and extreme temperatures caused by climate change lead to stress conditions that interfere with plant growth and crop productivity. Plants have been constantly evolving defense mechanisms to overcome these stress conditions, such as high temperatures, drought, high salinity, and cold. Osmotic stress, caused by water deficits, also affects plant growth and causes reduced crop yields. Among osmotic stresses, drought stress is closely related to stomatal adjustment and is one of the major abiotic stresses (Golldack et al., 2014; Lee and Luan, 2012). When plants are exposed to drought conditions, closure of stomata in response to abscisic acid (ABA) occurs to minimize water loss (Golldack et al., 2014; Lee and Luan, 2012).

ABA is a key hormone in plant responses to abiotic stress, especially drought stress (Osakabe et al., 2014), as it modulates several stress-related genes, including protein kinases, phosphatases, transcription factors, and ion channels involved in osmotic adjustment (Sirichandra et al., 2009). However, although previous studies have elucidated aspects of the ABA signaling pathway from the perspective of plant cells, the exact mechanism of the ABA signaling pathway remains unclear (Lee et al., 2009; Lim et al., 2015a; Ma et al., 2009; Park et al., 2009). The three major components involved in ABA-induced stomatal closure are 
the pyrabactin resistance/pyrabactin resistance-like/regulatory component of ABA receptor (PYR/PYL/RCAR) proteins, sucrose non-fermenting 1-related subfamily 2 (SnRK2) protein kinases, and type $2 \mathrm{C}$ protein phosphatases (PP2Cs) (Lee and Luan, 2012; Ma et al., 2009; Park et al., 2009). PYR/PYL/RCAR ABA receptors initiate the ABA signaling pathway in cells and transfer the signal to clade A PP2Cs, which negatively modulate the ABA signaling pathway, and to SnRK2s protein kinases, which positively modulate the ABA signaling pathway (Lee and Luan, 2012). PYR/PYL/RCAR ABA receptors interact with clade A PP2Cs (Szostkiewicz et al., 2010), interrupting the interaction between clade A PP2Cs and SnRK2s. Released SnRK2 protein kinases enhance specific gene expression and activate ion channels and transcription factors via phosphorylation of target proteins (Lim et al., 2015a; Lee and Luan, 2012; Lee et al., 2009).

Additionally, ABA decreases the turgor pressure in guard cells by modifying the activities of several ion channels (Schroeder et al., 2001). SLAC1 and shaker type $\mathrm{K}^{+}$ channels are ABA-modulated ion channels that regulate anion and cation fluxes across the plasma membrane and tonoplast, which adjusts stomatal apertures (Lebaudy et al., 2010; Lemtiri-Chlieh and MacRobbie, 1994; Negi et al., 2008; Schroeder and Hagiwara, 1989; Schroeder et al., 1987; Vahisalu et al., 2008). In Arabidopsis, the shaker type $\mathrm{K}^{+}$channel is a voltage-gated channel composed of nine genes (Hedrich, 2012). The structure of the shaker type $\mathrm{K}^{+}$channel consists of six transmembrane domains and $\mathrm{N}$-terminal/C-terminal regions in the cytoplasm. The $\mathrm{C}$-terminal region is involved in protein-protein interactions and channel activity and targeting (Dreyer and Blatt, 2009; Nieves-Cordones et al., 2014; Pilot et al., 2003). Among the nine channels, KAT1, KAT2, AKT1, AKT2, and GORK are expressed in guard cells (Dreyer and Blatt, 2009; Lefoulon et al., 2016). It has been widely recognized that the anion efflux of SLAC1 activates GORK channels in guard cells, thus causing stomatal closure. Moreover, PP2C activity modulates stomatal aperture as it controls SLAC1 and GORK activity (Lefoulon et al., 2016).

Moreover, studies have reported that the RCAR2 ABA receptor positively regulates the ABA signaling pathway, whereas the clade A protein phosphatase PP2CA negatively regulates the $\mathrm{ABA}$ signaling pathway by modulating the SLAC1 anion channel activity (Lee et al., 2009, 2013). Additionally, these studies have also demonstrated that RCAR2 is associated with drought tolerance. In this study, we isolated the Arabidopsis GROK channel that interacts with PP2CA; we also isolated two T-DNA insertion mutants of GORK1 that are hyposensitive to ABA, high salin- ity, and osmotic stress in seeds and seedlings. Our findings indicate that GORK channels modulate ABA-induced stress responses.

\section{Materials and Methods}

Plant growth conditions. Seeds of Arabidopsis (Arabidopsis thaliana, ecotype Col-0) were grown in soil composed of equal parts of sterilized sand, loam soil, and a compost mixture of peat moss, perlite, and vermiculite $(5: 3: 2, \mathrm{v} / \mathrm{v} / \mathrm{v})$. Seeds were grown under white fluorescent light $(130 \mu \mathrm{mol}$ photons $/ \mathrm{m}^{2} / \mathrm{s}$ ) at $24 \pm 1^{\circ} \mathrm{C}$. For the germination and seedling growth experiment, Arabidopsis seeds were grown on Murashige and Skoog (MS) media (Duchefa Biochemie, Haarlem, Netherlands) supplemented with $1 \%$ sucrose in a growth chamber at $24 \pm 1{ }^{\circ} \mathrm{C}$ and under a 16-h light/8$\mathrm{h}$ dark period. Seeds were vernalized at $4^{\circ} \mathrm{C}$ for 2 days to synchronize germination.

Germination and seedling growth. To examine germination, primary root growth, and cotyledon greening, 200 seeds of wild-type plants, gork and $p p 2 c a$ mutant plants were plated on MS agar medium containing $\mathrm{ABA}, \mathrm{NaCl}$, and mannitol. Germination was determined by radicle emergence. Primary root growth was measured 7 days after plating, and green cotyledons were measured 10 days after plating. Plates were placed in a growth chamber at $24^{\circ} \mathrm{C}$ for a 16-h light/8-h dark cycle.

RNA isolation and reverse transcription PCR (RTPCR) analysis. Total RNA isolation and RT-PCR assays were conducted as described by Lim et al. (2015b), using leaf tissues of wild-type and gork mutant plants. To synthesize cDNA, we used $1 \mu \mathrm{g}$ of the total RNA and a Transcript First Strand cDNA Synthesis kit (Roche, Indianapolis, IN, USA), according to the manufacturer's protocol. For the RT-PCR assay, the cDNA was amplified using the iQ SYBR Green Supermix (Bio-Rad, Hercules, CA, USA) with GORK specific primers (F: CAGGAAACTTCCTTTGCACGAC; R: TCAAATGAATCATCGGAAAAATC). The AtACT8 gene was used for internal control.

Subcellular localization of GORK and PP2CA. For the subcellular localization analysis, we used full-length coding regions of GORK and PP2CA without the stop codon and p326GFP vector. To express each transient construct, Agrobacterium tumefaciens strain GV3101 was infiltrated to epidermal cells of Nicotiana benthamiana p19 strain (1:1 ratio; $\mathrm{OD}_{600}=0.5$ ). Four days after such transient expression, tobacco leaves were cut and epidermal cells were 
observed with a confocal microscope (510 UV/Vis Meta, Zeiss, Oberkochen, Germany).

Yeast two-hybrid analysis. To verify the interaction between GORK and PP2CA, we used a yeast two-hybrid (Y2H) analysis (Lee et al., 2007). The C-terminal region of GORK and the full-length cDNA of PP2CA were inserted into pGBKT7 and pGADT7 vectors, respectively, and transformed into the AH109 yeast strain using the lithium acetate-mediated method (Ito et al., 1983). To evaluate the interaction between GORK and PP2CA, yeast cells containing each construct were spotted on selection media (SC-adenine-histidine-leucine-tryptophan) and grown for 4 days.

Bimolecular fluorescence complementation (BiFC) analysis. $\mathrm{BiFC}$ analysis was conducted as described previously (Lim and Lee, 2016). To generate BiFC constructs, open reading frames without a stop codon for GORK and PP2CA were inserted into Pro35S:SPYNE and Pro35S:SPYCE vectors, respectively (Waadt et al., 2008). To express each transient construct, Agrobacterium tumefaciens strain GV3101 was infiltrated into epidermal cells of $N$. benthamiana $\left(\mathrm{OD}_{600}=0.5\right)$. Four days after agroinfil- tration, tobacco leaves were cut and epidermal cells were observed with a confocal microscope (510 UV/Vis Meta, Zeiss).

\section{Results and Discussion}

Reduced sensitivity of gork mutants in response to ABA. Previous studies have shown that GORK is involved in stomatal closure and water loss by transpiration (Hosy et al., 2003; Lefoulon et al., 2016). Even though it is widely recognized that clade A PP2Cs negatively modulates GORK channel activity and that CPK3 positively modulates GORK channel activity (Corratgé-Faillie et al., 2017; Lefoulon et al., 2016), the biological role of GORK in early stages of a plant's life cycle remains unclear. To investigate the biological functions of GORK in seeds and seedlings, we identified T-DNA insertion mutants gork-1 (SALK_082258) and gork-2 (SALK_144737) as knockout alleles (Fig. 1A). First, we performed RT-PCR analysis to verify the knockout of gork mutants (Fig. 1B). GORK transcripts were detected in wild-type plants but not in gork mutants. The identified gork mutants showed no obvious phenotype under normal growth conditions (Fig. 1C). However, growth of wild-type and gork mutant plants was

A

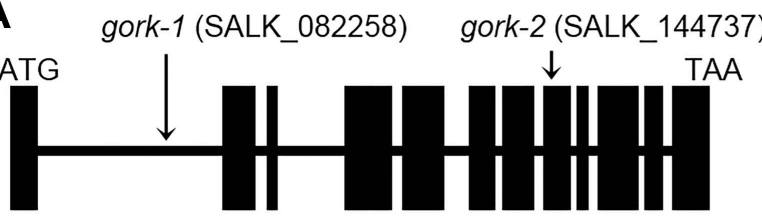

B
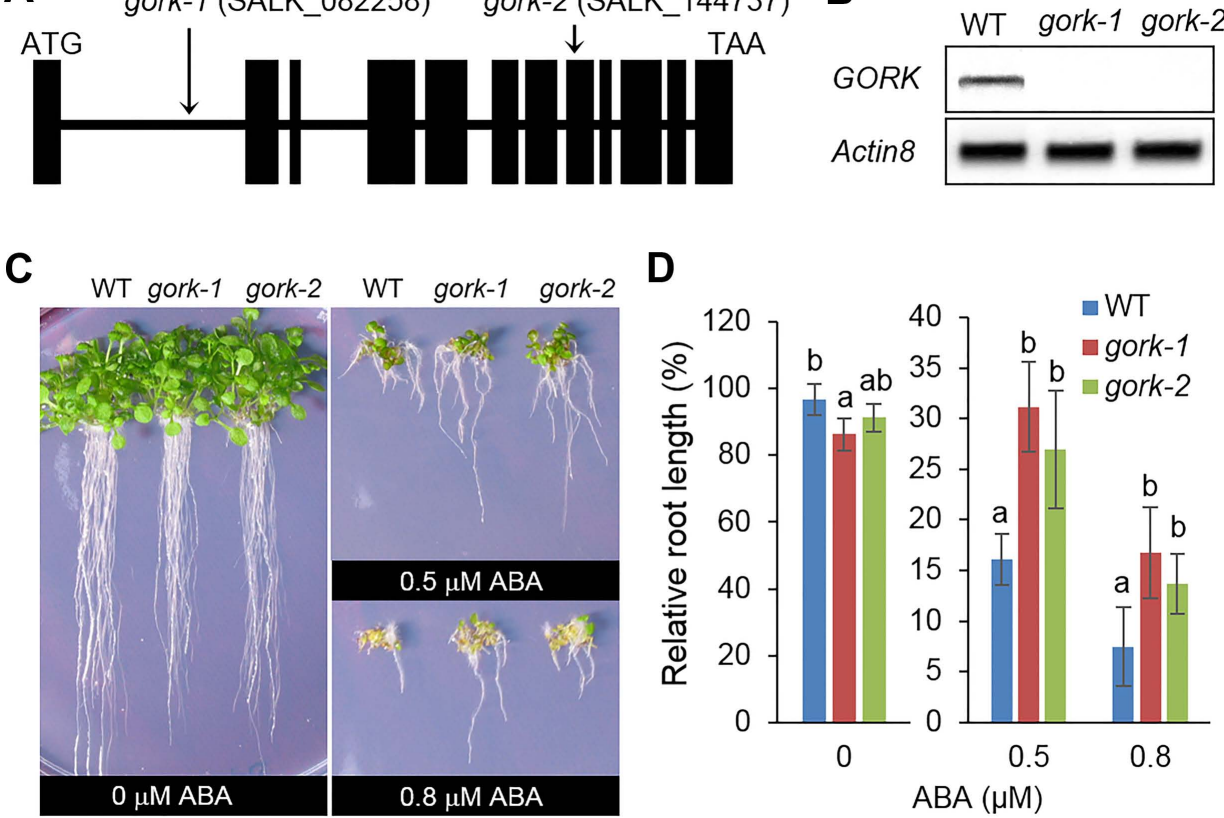

Fig. 1. Decreased sensitivity of gork mutants to abscisic acid (ABA). (A) Schematic diagram of the T-DNA insertion sites within the genomic DNA sequence of the GORK gene. Boxes refer to exons and bars to introns. (B) Relative expression of the GORK gene in wild-type (WT) and gork mutant plants. (C, D) Inhibition of primary root growth of WT and gork mutant plants in the presence of ABA. Seedlings were grown vertically in $0.5 \times$ Murashige and Skoog containing 0.5 and $0.8 \mu \mathrm{M}$ ABA. After 12 days, images were taken (C) and root lengths of each plant were measured (D). Data represent the mean $\pm \mathrm{SE}$ of three independent experiments. Different letters indicate significant difference between WT and mutant plants (ANOVA; $P<0.05$ ). 
A

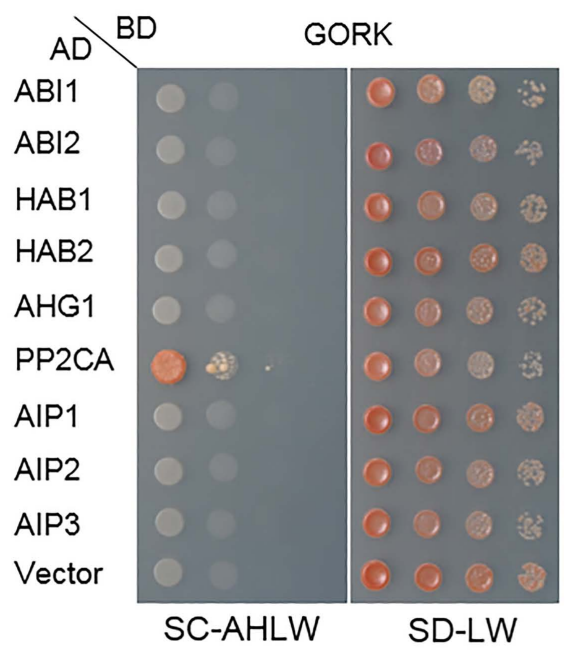

C

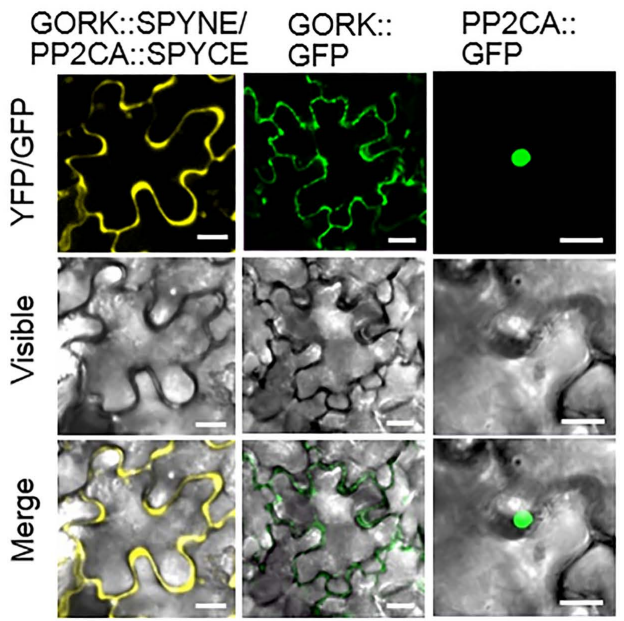

B

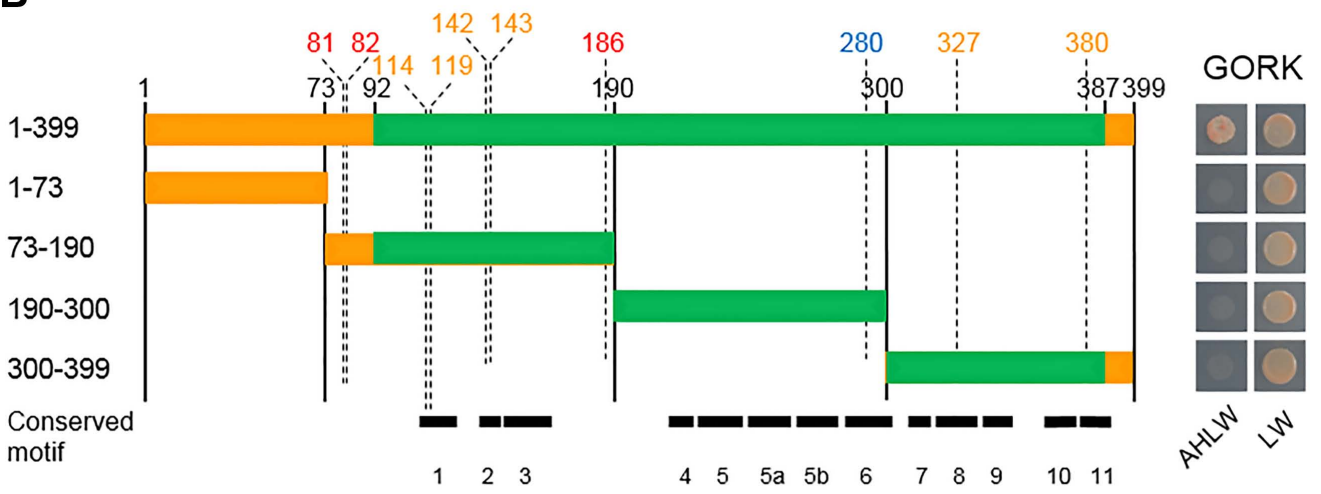

Orange: active site; A binuclear Mn2- ion site

Red: ubiquitinated site

Blue: The 'lock' site stabilizes the complex made of PP2C, ABA and PYR/PYL/RCAR receptor by keeping receptor 'gate' and 'latch' loops in closed positions

Black box: conserved motif

Fig. 2. Physical interactions between GORK and PP2CA. (A) Yeast-2 hybrid (Y2H) assay of the interactions between GORK and PP2Cs. Growth on SC-ALTW was used as an indicator of the interaction (left row). Growth on SC-LW was used as the control (right row). (B) Y2H assay of the interactions between GORK and PP2CA fragment domains. (C) Bimolecular fluorescence complementation assay of the interaction between GORK and PP2CA and their subcellular location in leaves of Nicotiana benthamiana. The yellow fluorescence indicates an interaction between the indicated partner proteins. The images were obtained from the YFP channel, bright field, or a merged picture of the two. Scale bars $=20 \mu \mathrm{m}$.

inhibited in the presence of 0.5 and $0.8 \mu \mathrm{M}$ of ABA. Additionally, primary root lengths of gork mutants were significantly longer than that of wild-type plants (Fig. 1C and D).

Interaction between GORK and PP2CA. Earlier, we demonstrated that PP2CA inhibits the activity of SnRK2.6 kinase, which consequently inhibits the SLAC1 anion channel (Lee et al., 2009, 2013). We conducted a Y2H assay to isolate the clade A PP2C interacting with GORK.
We used the GORK C-terminal region as bait and clade A PP2Cs as prey. We performed a one-by-one $\mathrm{Y} 2 \mathrm{H}$ assay with clade A PP2Cs (Fig. 2A); specifically, the yeast with GORK and PP2CA grew on the selective media, suggesting that GORK interacts with PP2CA. It has been demonstrated that the domain of clade A PP2Cs, including specific amino acids, plays an important role in the interaction with RCARs and SnRK2 kinases (Fuchs et al., 2013); however, little is known about the interaction with GORK. 
To elucidate which domain is important in the interaction between GORK and PP2CA, we generated truncated forms of PP2CA and performed $\mathrm{Y} 2 \mathrm{H}$. Growth of yeast with GORK was only detected with full-length PP2CA (Fig. 2B), indicating that full-length PP2CA is necessary for the interaction to occur. We used $\mathrm{BiFC}$ analysis to determine where the interaction complex was located inside the cell and green fluorescent protein (GFP) analysis to determine where each protein was located inside the cell (Fig. 2C). Co-expression of GORK-SPYNE with PP2CA-SPYCE was detected as yellow fluorescence predominantly in plasma membrane. The GFP signals of GORK-GFP and PP2CA-GFP were generated in the plasma membrane and nucleus, respectively. Such results agree with the Y2H analysis and indicate a physical interaction between GORK and PP2CA. In addition, it also suggests that GORK and PP2CA are both targeted at the plasma membrane, where they regulate $\mathrm{K}^{+}$efflux thus causing stomatal closure (Corratgé-Faillie et al., 2017; Lefoulon et al., 2016).

ABA sensitivity of GORK and PP2CA mutants. PP2CA has been reported as one of the core ABA signaling component, where it acts as a negative $\mathrm{ABA}$ regulator (Baek et al., 2018; Lee et al., 2013; Lim et al., 2015a). To investigate the in vivo function of GORK in response to ABA, we compared the germination and seedling growth of wildtype plants, gork and pp2ca mutant plants (Fig. 3). In the absence of exogenous ABA, we did not find differences between the phenotypes in relation to seed germination and seedling development. However, when grown on media containing ABA, gork mutants showed significantly higher germination in comparison to wild-type plants, whereas pp2ca mutants showed significantly lower germination rate than wild-type plants (Fig. 3A). We also compared primary root growth and seedling establishment of wild-type, gork, and pp2ca mutant plants (Fig. 3B-D). ABA-induced inhibition of primary root growth in gork mutants was significantly lower in comparison to wild-type and $p p 2 c a$ plants (Fig. 3B). Additionally, the number of green cotyledons in gork mutant plants was significantly higher in comparison to wild-type plants; whereas in $p p 2 c a$ mutants, the number of green cotyledons was significantly lower in comparison to wild-type plants (Fig. 3C and D). These results suggest that knockout of GORK decreases sensitivity to ABA during germination, which leads to reduced primary root growth and hinders seedling establishment.

Abiotic stress responses of gork and $p p 2 c a$ mutants. ABA signal and the abiotic stress response share common signaling components (Lim et al., 2015a; Sah et al., 2016).
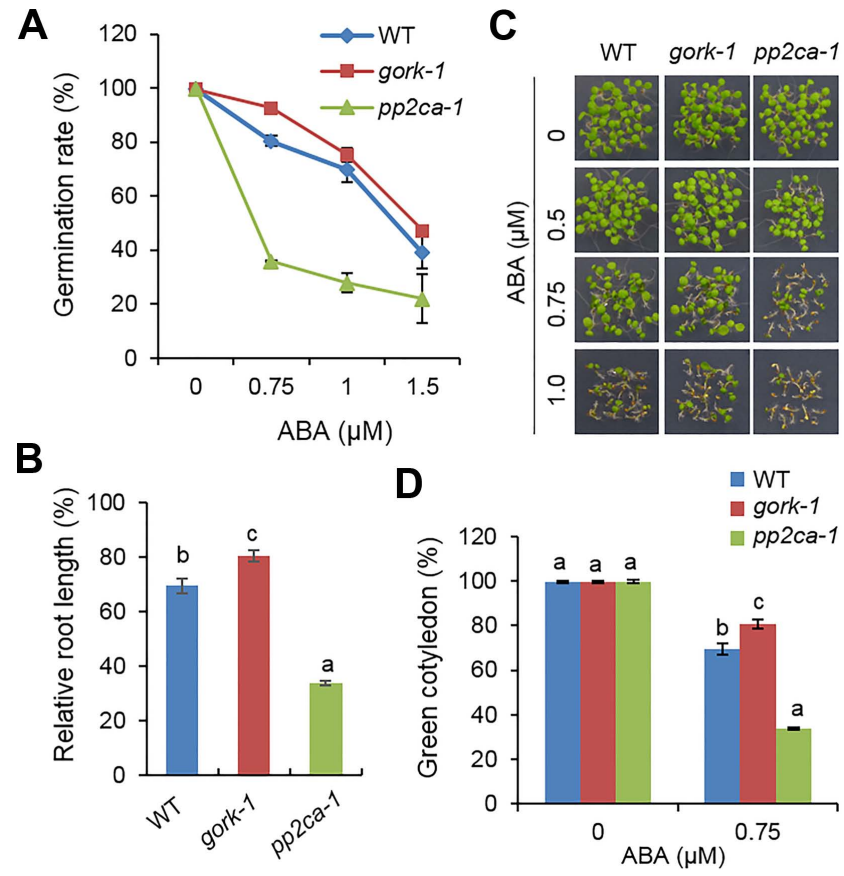

Fig. 3. Abscisic acid (ABA) sensitivity of gork and pp2ca mutants. (A) Germination of wild-type (WT), gork and pp2ca mutant plants on $0.5 \times$ Murashige and Skoog (MS) media containing different concentrations of ABA. Germination was determined by radicle emergence and was counted 3 days after plating. (B) Inhibition of primary root growth by ABA in WT, gork, and pp2ca mutant plants. Seedlings were grown vertically in $0.5 \times \mathrm{MS}$ containing $0.75 \mu \mathrm{M}$ of ABA for 7 days. (C, D) Number of green cotyledons in WT, gork, and pp2ca mutant plants in response to ABA. Representative photographs were taken at 10 days after plating (C), and the number of seedlings with expanded cotyledons was counted (D). Values are the mean \pm SE from three independent experiments. Different letters indicate significant difference between WT and mutant plants (ANOVA; $P<0.05$ ).

We therefore investigated the involvement of GORK and PP2CA in plant responses to abiotic stress, including high salinity and osmotic stress (Figs. 4 and 5). First, we evaluated the plants response to high salinity by comparing different phenotypes (wild-type, gork and pp2ca mutant plants) treated with $\mathrm{NaCl}$ (Fig. 4). Under regular growth conditions, there was no significant difference between wild-type and mutant plants. However, in the presence of $\mathrm{NaCl}, p p 2 c a$ mutants showed significantly lower germination rate compared to wild-type and gork plants (Fig. 4A). Moreover, the number of green cotyledons was significantly higher in gork mutant plants and significantly lower in pp2ca mutant plants in comparison to wild-type plants (Fig. $4 \mathrm{~B}$ and $\mathrm{C})$.

To further examine whether gork and $p p 2 c a$ mutants showed different responses to osmotic stress, we measured 
A

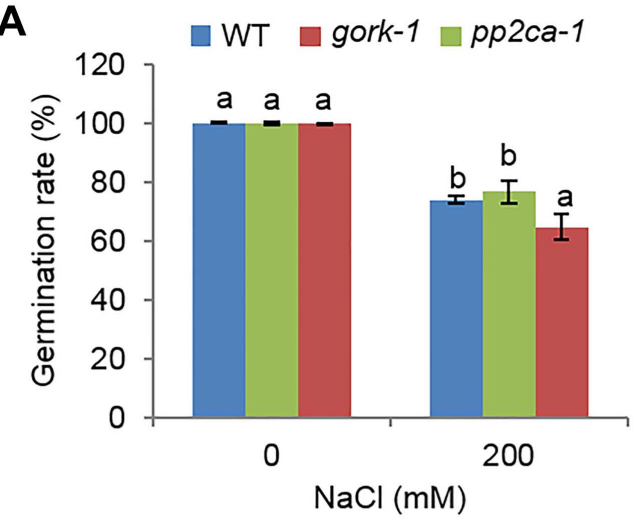

B

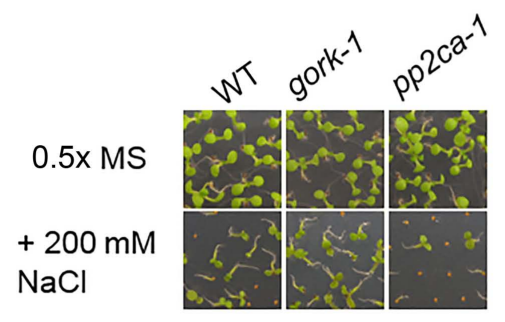

C

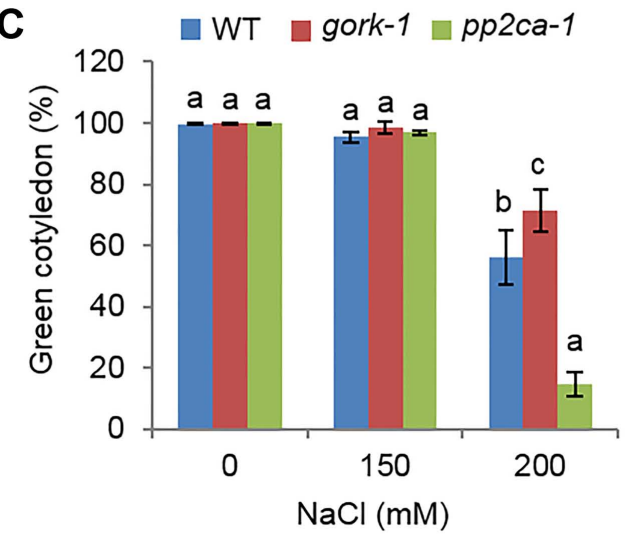

Fig. 4. Response of gork and pp2ca mutant plants to $\mathrm{NaCl}$. (A) Germination rates of wild-type (WT), gork, and pp2ca mutant plants on $0.5 \times$ Murashige and Skoog (MS) media containing 200 $\mathrm{mM}$ of $\mathrm{NaCl}$. Germination was determined by radicle emergence and was evaluated 9 days after plating. $(B, C)$ Number of green cotyledon in WT, gork, and pp2ca mutant plants in response to $\mathrm{NaCl}$. Representative photographs were taken at 10 days after plating (B), and the number of seedlings with expanded cotyledons was counted $(\mathrm{C})$. Values are the mean $\pm \mathrm{SE}$ from three independent experiments. Different letters indicate significant difference between WT and mutant plants (ANOVA, $P<0.05$ ).

germination and the number of green cotyledons in seeds treated with mannitol (Fig. 5). In contrast to the ABA and $\mathrm{NaCl}$ treatments, seed germination of gork and pp2ca mutants did not differ from that of wild-type plants (Fig. 5A). However, the number of green cotyledons was significantly higher in gork mutants and significantly lower in $p p 2 c a$
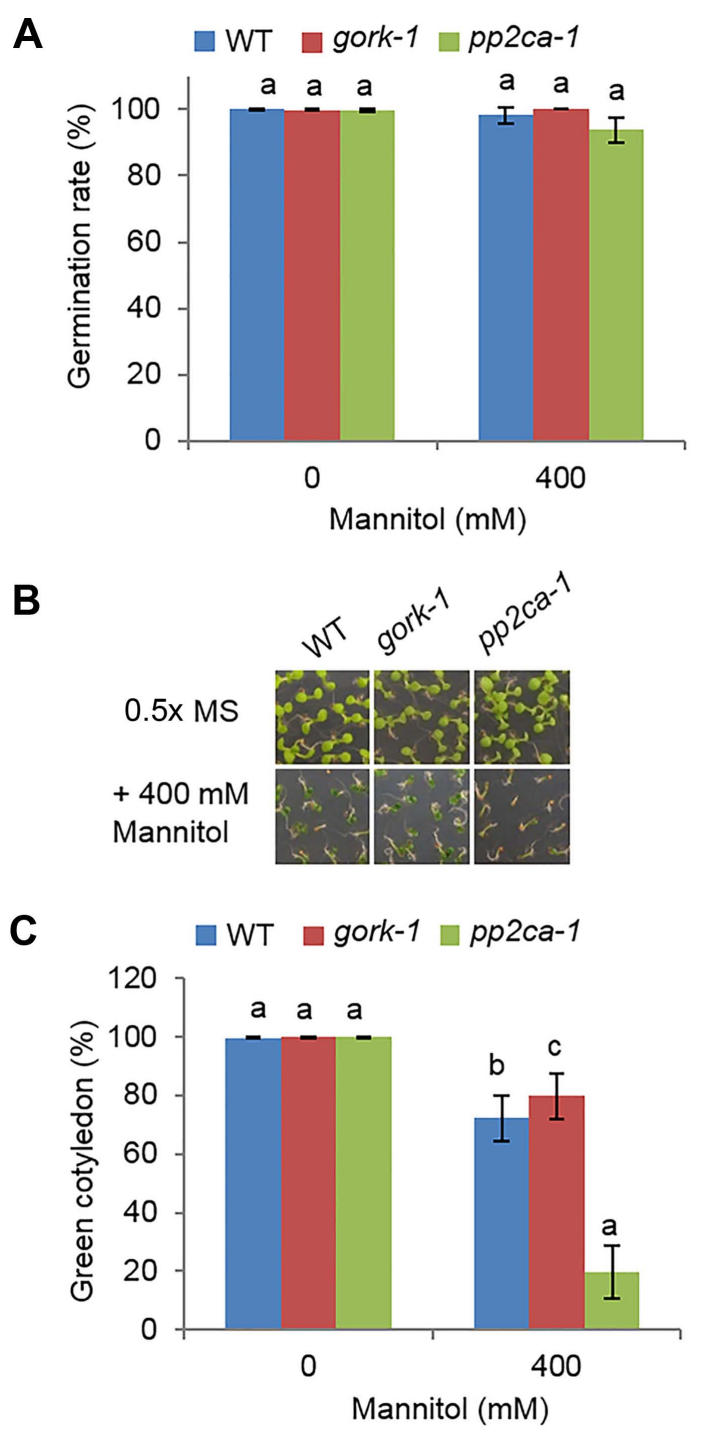

Fig. 5. Response of gork and pp2ca mutant plants to mannitol. (A) Germination rates of wild-type (WT), gork, and pp2ca mutant plants on $0.5 \times$ Murashige and Skoog media containing $400 \mathrm{mM}$ of mannitol. Germination was determined by radicle emergence and was evaluated 9 days after plating. (B, C) Number of green cotyledons in WT, gork, and pp2ca mutant plants in response to mannitol. Representative photographs were taken at 10 days after plating (B), and the number of seedlings with expanded cotyledons was counted $(C)$. Values are the mean \pm SE from three independent experiments. Different letters indicate significant difference between WT and mutant plants (ANOVA; $P<0.05$ ).

mutants in comparison to the number of green cotyledons found on wild-type plants (Fig. 5B and C). These results indicate that both GORK and PP2CA affect the plant responses to high salinity and osmotic stress. Together, these results are consistent with earlier studies and with the genetic analyses performed. In conclusion, the shaker type 
$\mathrm{K}^{+}$channel GORK is related to the ABA response as it interacts with clade A PP2C, affecting the plant's response to high salinity and osmotic stress. However, gork mutants do not appear to be related in regulating turgor pressure in guard cells in germination and seedling growth. Identification of additional regulators of GORK, such as other kinases or phosphatases, will help clarify the exact functions of GORK in the ABA signaling pathway in seeds and seedlings.

\section{Acknowledgments}

This work was supported by a grant from the "Next-Generation BioGreen 21 Program for Agriculture \& Technology Development (Project No. PJ01367603)," Rural Development Administration, and by the National Research Foundation of Korea (NRF) grant funded by the Korea Government (MSIT) (No. 2017R1D1A1B03031961), Republic of Korea.

\section{References}

Baek, W., Lim, C. W. and Lee, S. C. 2018. A DEAD-box RNA helicase, RH8, is critical for regulation of ABA signalling and the drought stress response via inhibition of PP2CA activity. Plant Cell Environ. 41:1593-1604.

Corratgé-Faillie, C., Ronzier, E., Sanchez, F., Prado, K., Kim, J.H., Lanciano, S., Leonhardt, N., Lacombe, B. and Xiong, T. C. 2017. The Arabidopsis guard cell outward potassium channel GORK is regulated by CPK33. FEBS Lett. 591:1982-1992.

Dreyer, I. and Blatt, M. R. 2009. What makes a gate? The ins and outs of $\mathrm{Kv}$-like $\mathrm{K}^{+}$channels in plants. Trends Plant Sci. 14:383-390.

Fuchs, S., Grill, E., Meskiene, I. and Schweighofer, A. 2013. Type 2C protein phosphatases in plants. FEBS J. 280:681693.

Golldack, D., Li, C., Mohan, H. and Probst, N. 2014. Tolerance to drought and salt stress in plants: unraveling the signaling networks. Front. Plant Sci. 5:151.

Hedrich, R. 2012. Ion channels in plants. Physiol. Rev. 92:17771811.

Hosy, E., Vavasseur, A., Mouline, K., Dreyer, I., Gaymard, F., Porée, F., Boucherez, J., Lebaudy, A., Bouchez, D., Very, A.A., Simonneau, T., Thibaud, J.-B. and Sentenac, H. 2003. The Arabidopsis outward $\mathrm{K}^{+}$channel GORK is involved in regulation of stomatal movements and plant transpiration. Proc. Natl. Acad. Sci. U. S. A. 100:5549-5554.

Ito, H., Fukuda, Y., Murata, K. and Kimura, A. 1983. Transformation of intact yeast cells treated with alkali cations. J. Bacteriol. 153:163-168.

Lebaudy, A., Pascaud, F., Véry, A.-A., Alcon, C., Dreyer, I., Thibaud, J.-B. and Lacombe, B. 2010. Preferential KAT1-
KAT2 heteromerization determines inward $\mathrm{K}^{+}$current properties in Arabidopsis guard cells. J. Biol. Chem. 285:62656274.

Lee, S. C., Lan, W., Buchanan, B. B. and Luan, S. 2009. A protein kinase-phosphatase pair interacts with an ion channel to regulate ABA signaling in plant guard cells. Proc. Natl. Acad. Sci. U. S. A. 106:21419-21424.

Lee, S. C., Lan, W.-Z., Kim, B.-G., Li, L., Cheong, Y. H., Pandey, G. K., Lu, G., Buchanan, B. B. and Luan, S. 2007. A protein phosphorylation/dephosphorylation network regulates a plant potassium channel. Proc. Natl. Acad. Sci. U. S. A. 104:1595915964.

Lee, S. C., Lim, C. W., Lan, W., He, K. and Luan, S. 2013. ABA signaling in guard cells entails a dynamic protein-protein interaction relay from the PYL-RCAR family receptors to ion channels. Mol. Plant 6:528-538.

Lee, S. C. and Luan, S. 2012. ABA signal transduction at the crossroad of biotic and abiotic stress responses. Plant Cell Environ. 35:53-60.

Lefoulon, C., Boeglin, M., Moreau, B., Véry, A.-A., Szponarski, W., Dauzat, M., Michard, E., Gaillard, I. and Chérel, I. 2016. The Arabidopsis AtPP2CA protein phosphatase inhibits the GORK $\mathrm{K}^{+}$efflux channel and exerts a dominant suppressive effect on phosphomimetic-activating mutations. J. Biol. Chem. 291:6521-6533.

Lemtiri-Chlieh, F. and MacRobbie, E. A.1994. Role of calcium in the modulation of Vicia guard cell potassium channels by abscisic acid: a patch-clamp study. J. Membr. Biol. 137:99-107.

Lim, C. W., Baek, W., Jung, J., Kim, J.-H. and Lee, S. C. $2015 \mathrm{a}$. Function of ABA in stomatal defense against biotic and drought stresses. Int. J. Mol. Sci. 16:15251-15270.

Lim, C. W., Hwang, B. K. and Lee, S. C. 2015b. Functional roles of the pepper RING finger protein gene, CaRING1, in abscisic acid signaling and dehydration tolerance. Plant Mol. Biol. 89:143-156.

Lim, C. W. and Lee, S. C. 2016. Pepper protein phosphatase type 2C, CaADIP1 and its interacting partner CaRLP1 antagonistically regulate ABA signalling and drought response. Plant Cell Environ. 39:1559-1575.

Ma, Y., Szostkiewicz, I., Korte, A., Moes, D., Yang, Y., Christmann, A. and Grill, E. 2009. Regulators of PP2C phosphatase activity function as abscisic acid sensors. Science 324:10641068.

Negi, J., Matsuda, O., Nagasawa, T., Oba, Y., Takahashi, H., Kawai-Yamada, M., Uchimiya, H., Hashimoto, M. and Iba, K. 2008. $\mathrm{CO}_{2}$ regulator SLAC1 and its homologues are essential for anion homeostasis in plant cells. Nature 452:483-486.

Nieves-Cordones, M., Chavanieu, A., Jeanguenin, L., Alcon, C., Szponarski, W., Estaran, S., Chérel, I., Zimmermann, S., Sentenac, H. and Gaillard, I. 2014. Distinct amino acids in the C-linker domain of the Arabidopsis $\mathrm{K}^{+}$channel KAT2 determine its subcellular localization and activity at the plasma membrane. Plant Physiol. 164:1415-1429.

Osakabe, Y., Osakabe, K., Shinozaki, K. and Tran, L.-S. P. 2014. 
Response of plants to water stress. Front. Plant Sci. 5:86.

Park, S.-Y., Fung, P., Nishimura, N., Jensen, D. R., Fujii, H., Zhao, Y., Lumba, S., Santiago, J., Rodrigues, A., Chow, T.F. F., Alfred, S. E., Bonetta, D., Finkelstein, R., Provart, N. J., Desveaux, D., Rodriguez, P. L., McCourt, P., Zhu, J.-K., Schroeder, J. I., Volkman, B. F. and Cutler, S. R. 2009. Abscisic acid inhibits type $2 \mathrm{C}$ protein phosphatases via the PYR/ PYL family of START proteins. Science 324:1068-1071.

Pilot, G., Pratelli, R., Gaymard, F., Meyer, Y. and Sentenac, H. 2003. Five-group distribution of the Shaker-like $\mathrm{K}^{+}$channel family in higher plants. J. Mol. Evol. 56:418-434.

Sah, S. K., Reddy, K. R. and Li, J. 2016. Abscisic acid and abiotic stress tolerance in crop plants. Front. Plant Sci. 7:571.

Schroeder, J. I. and Hagiwara, S. 1989. Cytosolic calcium regulates ion channels in the plasma membrane of Vicia faba guard cells. Nature 338:427-430.

Schroeder, J. I., Kwak, J. M. and Allen, G. J. 2001. Guard cell abscisic acid signalling and engineering drought hardiness in plants. Nature 410:327-330.

Schroeder, J. I., Raschke, K. and Neher, E. 1987. Voltage depen- dence of $\mathrm{K}^{+}$channels in guard-cell protoplasts. Proc. Natl. Acad. Sci. U. S. A. 84:4108-4112.

Sirichandra, C., Wasilewska, A., Vlad, F., Valon, C. and Leung, J. 2009. The guard cell as a single-cell model towards understanding drought tolerance and abscisic acid action. J. Exp. Bot. 60:1439-1463.

Szostkiewicz, I., Richter, K., Kepka, M., Demmel, S., Ma, Y., Korte, A., Assaad, F. F., Christmann, A. and Grill, E. 2010. Closely related receptor complexes differ in their ABA selectivity and sensitivity. Plant J. 61:25-35.

Vahisalu, T., Kollist, H., Wang, Y.-F., Nishimura, N., Chan, W.-Y., Valerio, G., Lamminmäki, A., Brosché, M., Moldau, H., Desikan, R., Schroeder, J. I. and Kangasjärvi, J. 2008. SLAC1 is required for plant guard cell S-type anion channel function in stomatal signalling. Nature 452:487-491.

Waadt, R., Schmidt, L. K., Lohse, M., Hashimoto, K., Bock, R. and Kudla, J. 2008. Multicolor bimolecular fluorescence complementation reveals simultaneous formation of alternative CBL/CIPK complexes in planta. Plant J. 56:505-516. 